\title{
Semioses
}

https://doi.org/10.15202/1981996x.2017v11n3p38

\section{OBTENÇÃO DE PADRÃO ANALÍTICO DE RETINOL A PARTIR DE FÍGADO BOVINO POR CROMATOGRAFIA LÍQUIDA DE ALTA EFICIÊNCIA}

\author{
OBTAINING ANALYTICAL STANDARD OF RETINOL FROM BOVINE LIVER BY \\ HIGH PERFORMANCE LIQUID CHROMATOGRAPHY
}

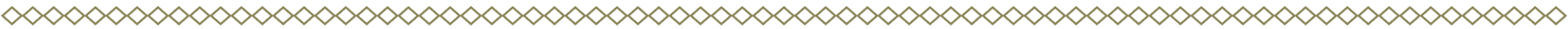

SABRINA DA SILVA DIAS

Doutora, Centro Universitário Estadual da Zona Oeste, Rio de Janeiro, RJ, Brasil sabrinadias@uezo.rj.gov.br

RONOEL LUIZ DE OLIVEIRA GODOY

Doutor, Embrapa Agroindústria de Alimentos, Rio de Janeiro, RJ, Brasil ronoel.godoy@embrapa.br

SIDNEY PACHECO

Doutor, Embrapa Agroindústria de Alimentos, Rio de Janeiro, RJ, Brasil sidney.pacheco@embrapa.br

MONALISA SANTANA COELHO DE JESUS

Mestre, Embrapa Agrobiologia, Seropédica, RJ, Brasil monalisa.coelho@embrapa.br

DANIEL FILISBERTO SCHULZ

Doutor, Instituto de Pesquisas Biomédicas do Hospital Naval Marcílio Dias, Marinha do Brasil Rio de Janeiro, RJ, Brasil danschulzms@gmail.com

ROSANA COLATINO SOARES REIS

Doutora, Universidade Federal Rural do Rio de Janeiro, Rio de Janeiro, RJ, Brasil rcolatino@ufrrj.br

CLAUDIO ROBERTO RIBEIRO BOBEDA

Doutor, Instituto Federal de Educação Ciência e Tecnologia do Rio de Janeiro, Rio de Janeiro, RJ, Brasil claudio.bobeda@ifrj.edu.br

\section{RESUMO}

Para a quantificação de substâncias de interesse, por Cromatografia Líquida de Alta Eficiência (CLAE), é necessário a obtenção de padrões analíticos confiáveis. Porém a aquisição destes padrões, com certificado de garantia, quase sempre depende de importação e apresenta custos elevados. As substâncias adquiridas ainda podem sofrer degradação, devido ao longo tempo de transporte e as condições inadequadas de armazenamento. A vitamina $\mathrm{A}$ ou retinol $\left(\mathrm{C}_{20} \mathrm{H}_{30} \mathrm{O}\right)$ é um álcool lipossolúvel encontrada apenas em fontes animais, em especial em áreas de armazenamento como o fígado ou associadas a gorduras como a do leite. O presente trabalho teve como objetivo isolar e quantificar retinol com alto teor de pureza a partir de fígado bovino para posterior utilização como padrão analítico. Após extração e hidrólise o retinol foi purificado do extrato de fígado por CLAE. A confirmação qualitativa foi realizada por Espectrometria de Massas de Alta Resolução (ESI-Q-TOF). Desta maneira foi possível a obtenção de retinol com $99 \%$ de pureza, demonstrando que a técnica utilizada possibilitou a preparação de padrão analítico para utilização em análises de CLAE. O processo reduz o risco de degradação da substância por condições inadequadas de transporte e manuseio, além de minimizar os custos dos procedimentos analíticos.

Palavras-chave: Conservação. Espermatozoide. Reprodução.

\section{ABSTRACT}

For the quantification of substances of interest, by 
High Performance Liquid Chromatography (HPLC), it is necessary to obtain reliable analytical standards. However, the acquisition of these standards, with warranty certificate, almost always depends on importation and presents high costs. Acquired substances may still be degraded due to long transport time and inadequate storage conditions. Vitamin A or retinol $\left(\mathrm{C}_{20} \mathrm{H}_{30} \mathrm{O}\right)$ is a lipid soluble alcohol found only in animal sour-ces, especially in storage areas such as the liver or as-sociated with fats such as milk. The objective of the present work was to isolate and quantify high purity retinol from bovine liver for later use as an analytical standard. After extraction and hydrolysis retinol was purified from liver extract by HPLC. The qualitative confirmation was performed by High Resolution Mass Spectrometry ESI-Q-TOF. In this way it was possible to obtain retinol with $99 \%$ purity, demonstrating that the technique used allowed the preparation of an analytical standard for use in HPLC. The process reduces the risk of substance degradation by inadequate trans-port and handling conditions, and minimizes the cost of analytical procedures.

Keywords: Carotenoid. Source. Isolation. Purifica-tion, Identification.

\section{INTRODUÇÃO}

A vitamina $\mathrm{A}$ (retinol $\mathrm{C}_{20} \mathrm{H}_{30} \mathrm{O}$ ) é uma substância constituída por 20 átomos de carbonos, contendo um anel beta-ionona e uma cadeia lateral isoprenóide, com um grupamento hidroxila na posição 1 (BURTINS e BRUNS, 2008; NILES, 2000). É um álcool, de massa molecular 286, lipossolúvel, com absorvância máxima em comprimento de onda de 325nm (MERCK, 1983). Essa vitamina só é encontrada em tecidos animais, ten-do como fontes principais o fígado, óleo de fígado de peixes, leites integrais e derivados, ovos e carnes de aves.

Uma das principais técnicas usadas para a quantificação de vitaminas lipossolúveis e seus isô-meros é a Cromatografia Líquida de Alta Eficiência (CLAE), devido à sua versatilidade, robustez e capa-cidade para analisar simultaneamente várias vitaminas lipossolúveis, com tempo reduzido de análise, com sensibilidade, especificidade e automação (PAIXÃO e STAMFORD, 2004). Estudos realizados por Zahar e Smith (1990) e Panfili, Manzi e Pizzoferato (1994) relatam a utilização dessa metodologia na determina-ção de monstrando a praticidade e confiabilidade da técnica para análise de vitamina $\mathrm{A}$.

Para a quantificação de substâncias de interesse, por HPLC é necessária a utilização de padrões analíticos confiáveis. Porém, a aquisição de padrões de alta pureza, possui custos elevados e quase sempre depende de importação, que em geral é morosa, e acaba trazendo incerteza na garantia da qualidade da substância adquirida. Em especial que pode estar comprometida devido a condições inadequadas de transporte e manuseio (PACHECO et al, 2010). Outro fator que pode comprometer as análises é a pureza dos padrões comerciais sintéticos, que pode conter impurezas provenientes da síntese.

Segundo a ANVISA (2010), um material com propriedades e estabilidade adequadas pode ser selecionado ou preparado em laboratório e usado como um padrão analítico. Assim, o presente trabalho teve como objetivo isolar e quantificar retinol com alto teor de pureza a partir de fígado bovino para posterior utilização como padrão analítico.

\section{METODOLOGIA}

\subsection{Equipamentos e reagentes}

Evaporador rotativo modelo Rotavapor-RE Buchi ${ }^{\circledR}$. Cromatográfo liquido de alta eficiência modular Waters composto por bomba 600, degaseificador de solvente em linha, injetor automático 717plus e detector de arranjo de fotodiodos modelo 996 e sistema de aquisição de dados com software Empower ${ }^{\circledR}$. Espectrofotômetro UV-1800, Shimadzu®. Cromatógrafo líquido de ultra-eficiência com detector de Espectrometria de massas (Acquity-Synapt ${ }^{\circledR}$ UPLC-MS/ MS- ESI/Q-TOF) Waters ${ }^{\circledR}$. KOH purificado - Tedia $\AA$, Pirogalol ACS - Vetec $\AA$, éter etílico, éter de petróleo, metanol e etanol, todos com grau HPLC - Tedia ${ }^{\circledR}$. Filtro de membrana $0,45 \mu \mathrm{m}$ de fluoreto de polivinilideno (PVDF) Millipore ${ }^{\circledR}$.

\subsection{Extração e Isolamento do padrão de retinol}

A extração de retinol foi realizada segundo método descrito por Campos (2003), com modificações. Aproximadamente $100 \mathrm{~g}$ de fígado bovino fresco foram triturados, e $20 \mathrm{~g}$ desse material foram pesados. A hidrólise alcalina do retinol foi realizada com $50 \mathrm{~mL}$ de solução de KOH:pirogalol:metanol 20:1:79 (m/v), durante 3 h, ao abrigo de luz. O hidrolisado foi então extraído em $100 \mathrm{~mL}$ de solução de éter de petróleo:éter 
etílico 20:80 (m/v), em duas etapas de 50mL. A fase etérea foi então separada em funil de separação e em seguida lavada com água MilliQ ${ }^{\circledR}$ até $\mathrm{pH}$ neutro. A fase etérea foi então evaporada em evaporador rotativo sob pressão reduzida, o resíduo foi então dissolvido com $5 \mathrm{~mL}$ de etanol e filtrado através de membrana $0,45 \mu \mathrm{m}$. A separação cromatográfica do retinol foi realizada no Laboratório de Cromatografia Líquida da Embrapa Agroindústria de Alimentos, utilizando coluna $\mathrm{C} 30(250 \times 4,6 \mathrm{~mm} \times 3 \mu \mathrm{m})-\mathrm{YMC} \AA$, a $33^{\circ} \mathrm{C}$, com eluição em modo isocrático de metanol:éter metil terc-butilico 80:20 (v/v) sob fluxo de 0,8mL/min (Pacheco et al., 2014). O volume de injeção foi de $15 \mu \mathrm{L}$, e o tempo de analise foi de 14 minutos seguidos de mais 14 minutos para eluição dos demais componentes da amostra, em especial dos carotenoides presentes. O retinol cromatograficamente separado foi coletado manualmente na saída do detector. Para se obter quantidades suficientes para a determinação de pureza e posterior utilização como padrão, foram realizadas injeções e coletas sucessivas.

\subsection{Quantificação espectrofotométrica do retinol isolado}

O retinol isolado foi quantificado por espectrofotometria em $325 \mathrm{~nm}$. O solvente utilizado foi o etanol absoluto e a absortividade molar para o cálculo da concentração foi $\varepsilon=1780$ (Merck, 1983).

\subsection{Determinação da pureza do retinol isolado}

A determinação da pureza do retinol isolado foi realizada por HPLC. A área do pico de retinol, em porcentagem relativa à soma das áreas de todos os picos presentes, foi considerada a pureza cromatográfica do padrão isolado.

\subsection{Espectrometria de massas do retinol isolado}

A confirmação qualitativa do retinol purificado foi realizada por Cromatografia Líquida de Ultra Eficiência com detector de massas de alta resolução. As condições cromatográficas foram: eluição isocrática de solução aquosa de ácido fórmico $0,1 \%$ sob fluxo de $0,25 \mathrm{~mL} / \mathrm{min}$. Coluna C18 (150mm x 2,1mm, 1,7 $\mu \mathrm{m})$ Waters ${ }^{\circledR}$ a $24^{\circ} \mathrm{C}$. Para a detecção foram utilizados os seguintes parâmetros: ionização por eletronebulização $(+)$, voltagem do capilar $4 \mathrm{kV}$ e do cone $25 \mathrm{~V}$, temperatura da fonte $100^{\circ} \mathrm{C}$ e fluxo de nitrogênio de dessolvatação $300 \mathrm{~L} / \mathrm{min}$ a $200^{\circ} \mathrm{C}$. Para confirmação da frag- mentação do retinol foi utilizado um padrão comercial de acetato de retinol. Este foi hidrolisado, da mesma maneira que a amostra, e então solubilizado em solução de metanol e éter metil terc-butílico 90:10 (v/v). Esta solução analisada por inserção direta no espectrômetro de massas sob os mesmos parâmetros descritos anteriormente.

\section{RESULTADOS e DISCUSSÃO}

\subsection{Extração e isolamento do retinol}

A identificação cromatográfica do retinol isolado foi feita através de comparação dos tempos de retenção e espectros de UV/Vis com padrão comercial de acetato de retinol hidrolisado, conforme Figura 1.

Rodriguez-Delgado e colaboradores (2002) analisaram retinol por cromatografia líquida, o espectro de absorção obtido, na faixa de comprimento de onda de 250 a $400 \mathrm{~nm}$ foi equivalente ao encontrado neste trabalho.

Figura 1 a) Cromatograma e espectro UV/Vis do retinol isolado de fígado bovino, b) Cromatograma e espectro UV/Vis do padrão comercial de acetato de retinol hidrolisado.
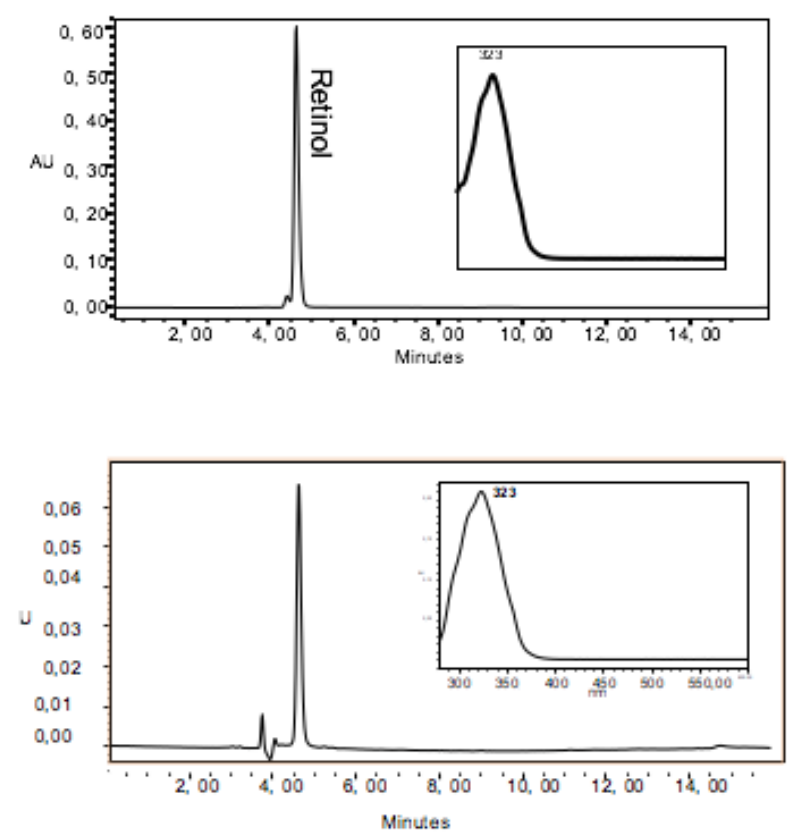

\subsection{Espectrometria de massas do retinol isolado}

A análise por CLUE-MS/MS- ESI/Q-TOF comprovou a presença de retinol no extrato de fígado bovino, como mostrado na Figura 2a. O espectro do pico com tR 1,67min mostra os fragmentos que carac- 
terizam o retinol: $\mathrm{m} / \mathrm{z} 269 ; 213,119$ e 95 .

Através da análise do padrão comercial de acetato de retinol hidrolisado (Figura $2 \mathrm{~b}$ ) pode-se caracterizar a fragmentação do retinol. Não é possível observar o sinal referente à molécula de retinol protonada ( $\mathrm{m} / \mathrm{z}$ 287), pois segundo Crotti et al (2006) durante a ionização por "electrospray" podem ocorrer reações ácido-base, complexação com cátions e ânions e/ou reações redox, dependendo das propriedades químicas da molécula, gerando três tipos de íons: íons moleculares, moléculas protonadas/desprotonadas e moléculas cationizadas ou anionizadas. Assim, após a formação dos íons provenientes do retinol em fase gasosa na fonte de eletronebulização, a molécula rapidamente sofre desidratação, gerando um fragmento positivo altamente estável pela deslocalização das ligações duplas da cadeia isoprenóide.

A presença do pico base m/z 269 foi utilizada para caracterizar o retinol (LI et al.,2005; WANG et al., 2001). E, nesse caso, a presença dos íons $\mathrm{m} / \mathrm{z} 213$, 177 e 95 corroboram a estrutura do retinol.

Em estudos realizados por Breemen et al (1998), a identificação do retinol nas amostras foi feita com base na presença do íon majoritário $\mathrm{m} / \mathrm{z} 269$, correspondendo ao retinol após perda de água. O mesmo íon foi observado em estudo realizado por Heudi, Trisconi e Blake (2004) analisando retinol em fórmulas infantis enriquecidas. Em trabalho realizado por Li et al (2005), novamente, o íon majoritário $\mathrm{m} / \mathrm{z} 269$ foi observado após perda de água $([\mathrm{m}+\mathrm{H})-\mathrm{H} 2 \mathrm{O}]+269)$.

Figura 2 a) Espectro de massas do retinol isolado de fígado bovino, obtido por CLUE-MS/MS- ESI/Q-TOF, b) Espectro de massas do padrão comercial de acetato de retinol hidrolisado obtido por inserção direta.
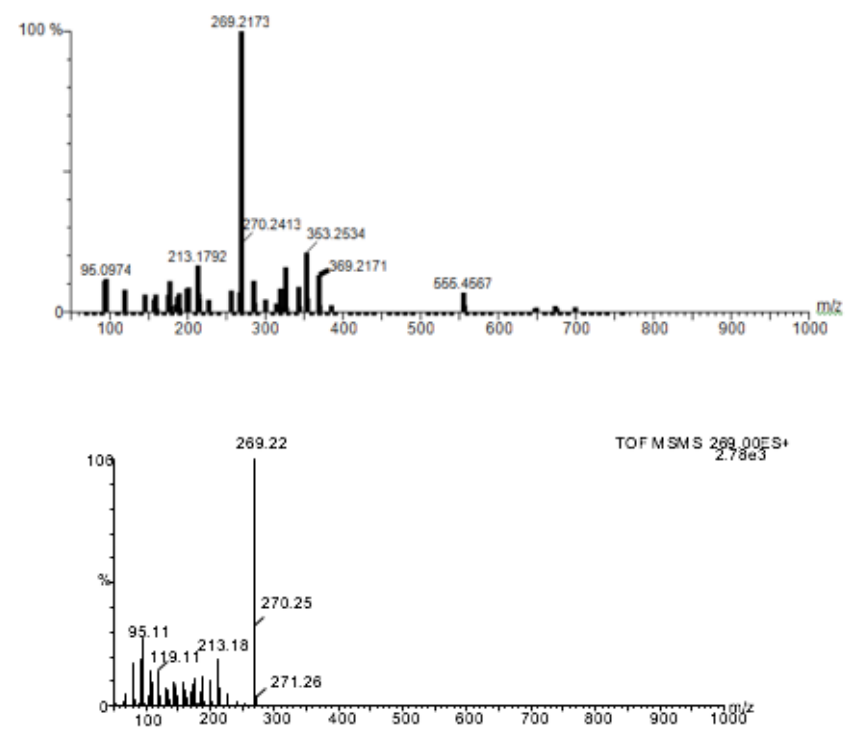

\subsection{Quantificação e determinação do teor de pureza do retinol isolado}

Após o isolamento, a concentração da solução obtida foi calculada através da absortividade molar e a leitura obtida por espectrofotometria a $325 \mathrm{~nm}$, resultando em $1,18 \mu \mathrm{g}$ de retinol por mililitro de solução. Com a separação cromatográfica e coleta manual do retinol hidrolisado e extraído de fígado bovino, foram obtidos $4 \mathrm{~mL}$ de solução de retinol com pureza de $99 \%$. quantidade suficiente para utilização como padrão analítico em análises cromatográficas.

\section{CONSIDERAÇÕES FINAIS}

Com a metodologia proposta neste trabalho, foi obtido padrão de retinol com $99 \%$ de pureza, isolado de fonte natural, demonstrando que a técnica utilizada possibilita a preparação de padrão analítico para utilização em análises por cromatografia líquida de alta eficiência. $\mathrm{O}$ método garante a qualidade das análises, pois reduz o risco de comprometimento do padrão analítico por condições inadequadas de transporte e manuseio, além de minimizar os custos dos procedimentos analíticos e a interferência de impurezas provenientes do processo de produção de padrão comercial.

Agradecimentos: Ao Conselho Nacional de Desenvolvimento Científico e Tecno $\neg$ lógico $(\mathrm{CNPq})$ pelo suporte financeiro.

\section{REFERÊNCIAS}

ANVISA, SENAI. Seleção, uso e interpretação de programas de ensaios de proficiência (EP) por laboratórios. Brasília(DF): ANVISA/SENAI; 2000.

BREEMEN, R. B.; NIKOLIC, D.; XU, X.; XIONG, Y.; LIESHOUT, M. V.; WEST, C. E.; SCHILLING, A. B. Development of a method for quantitation of retinol and retinyl palmitate in human serum using high-performance liquid chromatography-atmospheric pressure chemical ionization-mass spectrometry. Journal of chromatography A. n. 794, p. 245-251, 1998.

BURTIS, A. C., BRUNS, E. D. TIETZ. Fundamentos de Química Clínica, 6a ed. Rio de Janeiro(RJ): Elsevier; 2008. 
CAMPOS, J. M. Perfil dos níveis de vitaminas A e E em leites de doadoras primíparas e multíparas em bancos de leite humano [Dissertação de mestrado]. Recife (PE): Universidade Federal de Pernambuco; 2005.

CROTTI, A. E. M.; VESSECHI, R.; LOPES, J. L. C.; LOPES, N.P. Espectrometria de massas com ionização por "electrospray": Processos químicos envolvidos na formação de íons de substâncias de baixo peso molecular. Química Nova, v.29, n.2, p.287-292, 2006.

HEUDI, O.; TRISCONI, M. J.; BLAKE, C. J. Simultaneous quantification of vitaminas $\mathrm{A}, \mathrm{D} 3$, and $\mathrm{E}$ in fortified infant formulae by liquid chromatography-mas spectrometry. Journal of chromatography A. n. 1022, p.115-123, 2004.

LI, H.; TYNDALE, S.T.; HEATH, D. D.; LETCHER, R. J. Determination of carotenoids and all-trans-retinol in fish eggs by liquid chromatography-electrospray ionization-tandem mass spectrometry. Journal of Chromatography B. v. 816, p. 49-56, 2005.

MERCK \& CO. The Merck índex na encyclopedia of chemicals, drugs, and biologicals, 10a ed, USA(N.J.): Rahway; 1983.

NILES, R. M. Recent advances in the use of vitamin A (retinoids) in the prevention and treatment of câncer. Nutrition, n. 16, v.11/12, p. 1084-90, 2000.

PACHECO, S.; GODOY, R. L. O.; ARAUJO, M. C. P.; ROS, J. S.; BORGUIN, R. G.; FELBERG, I.; GOUVÊA, A. C. M. S. Preparo de Padrões analíticos de alta pureza usando cromatografia líquida de alta eficiência em escala analítica. $3^{\circ}$ Simpósio de Segurança alimentar -SBCTA-RS; Maio/junho de 2010; Florianópolis/ SC.

PACHECO, S.; PEIXOTO, F. M.;BORGUINI, R. G.; NASCIMENTO, L. S. M.; BOBEDA, C. R. R.; SANTIAGO, M. C. P. A.; GODOY, R. L. O. Microscale extraction method for HPLC carotenoid analysis in vegetable matrices. Sci. Agric., 71 (5), 416-419, 2014

PAIXÃO, J. A.; STAMFORD, T. L. M. Vitaminas lipossolúveis em alimentos - Uma abordagem analítica. Química Nova. v.27, n.1, p. 96-105. 2004. gh-performace liquid chromatographic method for the simultaneous determination of tocopherols, carotenes, and retinol and its geometric isomers in italian cheeses. Analyst, n.119, p.1161- 65, 1994.

RODRIGUEA-DELGADO, M. A.; DÍAZ-FLORES, E. J. F.; CALZADILLA, C. H.; ROMERO, C. D. Fast determination of retinol and $\square$-tocoferol in plasma by LC. Journal of pharmaceutical and biomedical analysis, n. 28, p. 991-7, 2002.

WANG, Y.; CHANG, W. Y.; PRIMS, G. S.; VAN BREEMEN, R. B. Simultaneous determination of all-trans, 9-cis, 13-cis retinoic acid and retinol in rat prostate using liquid chromatography - mass spectrometry. Journal of Mass Spectrometry, n. 36, v.8, p.882-888, 2001 .

ZAHAR, M.; SMITH, D. E. Vitamin A quantification in fluid dairy products: rapid method for vitamin A extration for high performace liquid chromatography. Journal Dairy Science, v. 73, p.3402-07, 1990.

PANFILI, G.; MANZI, P.; PIZZOFERRATO, L. Hi- 